\title{
The Effects of Progressive Resistance Training on Obstructed-Gait Tasks in Community-Living Older Adults
}

\author{
Ecosse L. Lamoureux, Aron Murphy, Anthony Sparrow, \\ and Robert $U$. Newton
}

\begin{abstract}
This study examined the effects of improved strength on an obstacle course (OC) simulating gait tasks commonly encountered by community-living older adults. Forty-five adults (mean age $68.2 \pm 1.5$ years) were randomly assigned to a control (10 women, 5 men) or an experimental group (EXP; 19 women, 10 men) and trained 3 days/week for 12 weeks. Using a 1-repetition-maximum (1$\mathrm{RM}$ ) method, 6 leg-strength measures were evaluated pre- and posttest. The times to walk an OC of 4 gait tasks (stepping over and across an obstacle, negotiating a raised surface, and foot targeting) set at 3 progressively challenging levels were also assessed. Significant Group $\times$ Time interactions were found on all 1-RM tests, with only EXP recording significant improvements $(124-147 \% ; p<.001)$. Strength gains in EXP were accompanied by significant improvements in the times to negotiate all gait stations and walk the entire OC $(6-15 \% ; p=.001-.014)$. This study showed that improving strength is an effective strategy to improve community locomotion, which might decrease the risks of falls in community-living older adults.
\end{abstract}

Key Words: dynamic strength, obstacle course, community-dwelling elderly, functional mobility

It has been unequivocally shown that resistance training can partially reverse age-related muscle weakness and atrophy (Charette et al., 1991; Fiatarone et al., 1990; Roman et al., 1993). The functional benefits of exercise-induced strength gains in older adults, though, have not been clearly established, with the research focusing on the effects of improved strength on unobstructed level walking (Chandler et al., 1998; Fiatarone et al., 1990, 1994; Hunter et al., 1995). Community-living older adults commonly encounter a range of locomotor challenges other than level walking, however, such as stepping over and across obstacles, negotiating raised surfaces, and foot targeting. To date there have been few investigations on the effectiveness of resistance training in obstructed gait in community-dwelling older

Lamoureux and Sparrow are with the School of Health Sciences, Deakin University, Melbourne, VIC, Australia. Murphy is with the school of Human Movement Studies, University of Technology, Sydney, NSW, Australia. Newton is with the Biomechanics Laboratory, Ball State University, Muncie, IN 47306. 
adults. This is probably because of the difficulty of reproducing a pertinent environmental condition in a laboratory setting.

An alternative to replicating real-life gait conditions could be to use an obstacle course simulating community locomotor challenges. Obstacle courses have been used previously with institutionalized older adults (Imms \& Eldholm, 1981), frail elderly participants (Brown, Sinacore, \& Host, 1995), and older participants with balance and mobility dysfunction (Means, 1996; Means, Rodell, \& O'Sullivan, 1996). The obstacle courses used in these studies were, however, set at one uniform level for all participants and included stations that did not adequately mirror challenging environmental gait tasks. An obstacle course normalized to an individual's stature and including gait tasks at progressively challenging levels could provide a more effective assessment of the effects of enhanced strength on real-life locomotion in community-living older adults. The aim of the experiment reported here was to determine the effect of strength training on performance on an obstacle course comprising gait tasks of progressive difficulty that simulated realworld locomotor challenges.

\section{Participants}

Adults age 60 years and over were invited to participate in the study. They were recruited from the suburbs surrounding Deakin University (Melbourne, Australia) via advertisements in the local newspapers. Telephone screenings initially eliminated those unsuitable for participation. Participants were required to be independent community-dwelling individuals, functionally mobile, with no serious cardiovascular disease, musculoskeletal disorders, neurological dysfunction, or uncontrolled chronic conditions. A physician screened the participants for medical conditions that would preclude exercise testing and resistance training. In addition, the participants were required to meet a prespecified criterion of low physical activity level, defined as exercise participation not exceeding 20 min once per week. This was determined by an in-house questionnaire. The final sample included 45 adults, who were randomly assigned to either a control (CON; $n=15 ; 10$ women, 5 men) or experimental group (EXP; $n=30 ; 19$ women, 11 men). All participants provided informed consent, and Deakin University Ethics Committee approval was obtained before the study was started.

\section{Measurements and Testing Procedures}

\section{ANTHROPOMETRIC ASSESSMENT}

Body mass $(\mathrm{kg})$, height $(\mathrm{cm})$, and leg and foot lengths $(\mathrm{cm})$ of all participants were assessed at pretest. Leg length was defined as the distance from the greater trochanter to the ground. Foot length was measured as the horizontal distance between the back of the Achilles heel and the distal end of the big toe.

\section{1-REPETITION-MAXIMUM TESTING}

Dynamic strength testing of the lower body muscle groups was assessed using a 1repetition-maximum (1-RM) method performed on custom-built pin-loaded weight 
machines. A 1-RM is defined as the maximal weight an individual can lift once through a specified range of motion. A 1-RM performance was assessed for the hipflexion and -extension, knee-extension and -flexion, and ankle plantar-flexion exercises. The maximum weight lifted for each exercise was recorded as the participant's 1-RM.

\section{PARTICIPANT POSITIONING FOR 1-RM TESTING}

Unilateral Hip Extension. Each participant was positioned sideways with the rotational axis of the machine and maintained an erect body posture and then flexed the hip joint until the superior aspect of the thigh was horizontal. As that position was held, the pad of the input arm of the machine was placed posterior to the knee joint with the knee flexed at $90^{\circ}$. The participant then extended the hip joint in a controlled movement until the superior aspect of the thigh reached a vertical position.

Unilateral Hip Flexion. The participant was positioned sideways with the rotational axis of the machine and maintained an erect body posture. The input arm of the machine was then positioned vertically with the pad resting just above the superior border of the patella. The participant then flexed the hip joint in a controlled movement until the superior aspect of the thigh reached a horizontal position.

Bilateral Knee Extension. The hip-angle position was individually determined by moving the back pad so that it allowed the knee to reach full extension from an original knee angle of $90^{\circ}$. Once the participant was comfortably seated, the arm was lowered vertically down until the pad rested on top of the ankle joint. From that position, the participant extended the knee joint until it reached full extension.

Bilateral Knee Flexion. The participant first lay on the bed of the machine with the superior border of the patella positioned a few centimeters past the edge of the bed. The input arm was then lowered and adjusted so that the pad of the arm rested just above the Achilles heel. From that position, the participant flexed the knee until the input arm reached vertical position.

Bilateral Ankle Plantar Flexion. After the participant assumed a seated position with the knees flexed at $90^{\circ}$, the T-shaped input arm was secured above the superior border of the patella. From that position, the participant extended the ankle joint and finished the movement on the balls of the feet.

\section{PROTOCOL FOR 1-RM TESTING}

Each participant was familiarized with the procedures of 1-RM testing using submaximal and maximal performances a week before testing. On a testing day, the participant underwent a 10-min standardized warm-up routine consisting of stationary cycling or comfortable walking and stretching the lower body muscle groups. Then, starting with a weight used in the preliminary familiarization sessions, the participant attempted lifts with gradually increasing weights (10\% at first, decreasing to $5 \%$ and $2.5 \%$ as difficulty became evident). Successive attempts were undertaken with a 2-min rest between attempts until the participant could not successfully complete the lift. Verbal encouragement was provided. A maximum of three to five 
trials was needed to determine the best 1-RM for each exercise (as found in similar studies, e.g., Charette et al., 1991; Hunter et al., 1995; Morganti et al., 1995).

All participants completed the strength-assessment tests. No injuries were reported among the participants, but there was some delayed-onset muscle soreness.

\section{LAYOUT OF THE OBSTACLE COURSE}

Gait tasks simulating environmental conditions were assessed on an obstacle course (OC) set up inside a gymnasium (Figure 1). The OC comprised four gait tasks at three progressively challenging levels: low, medium, and high challenges (Levels 1,2 , and 3, respectively). The gait tasks included stepping over obstacles of height and width, negotiating a raised surface, and foot targeting. The gait tasks were set

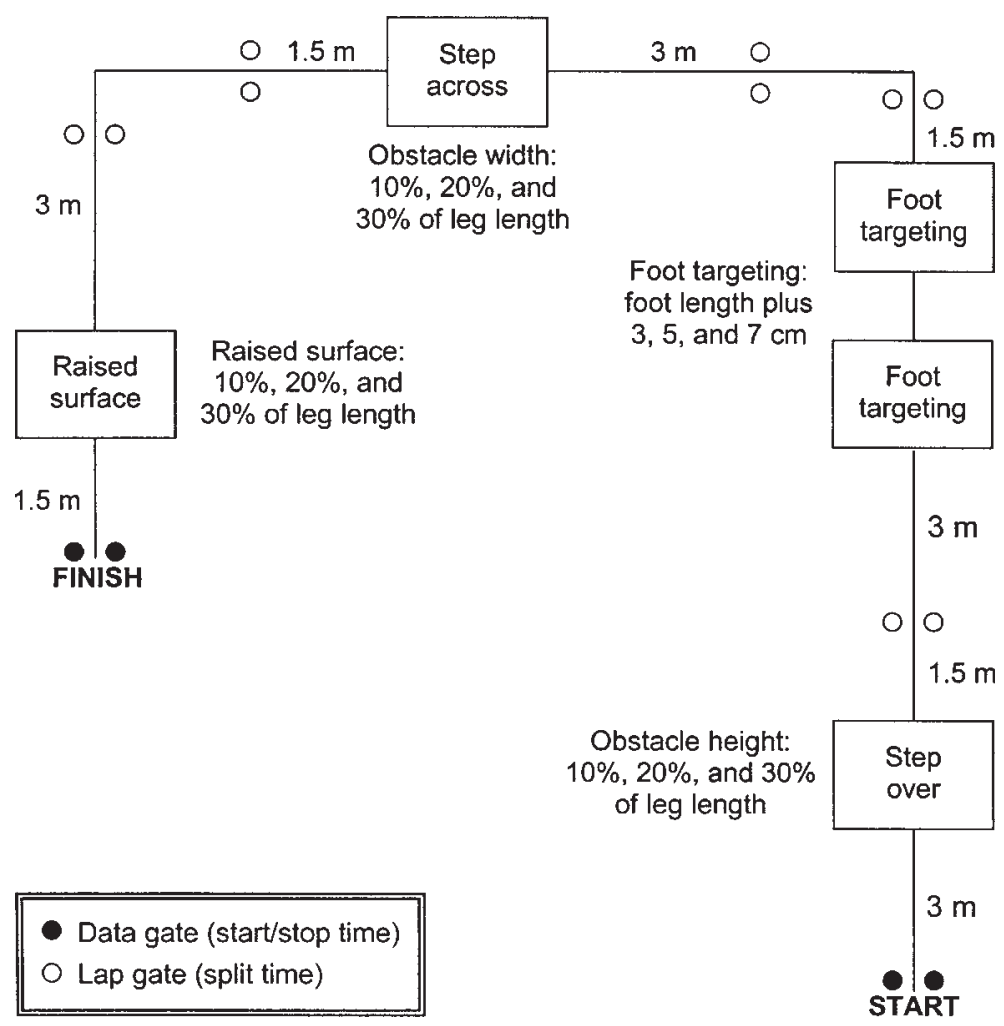

Figure 1. Layout of the obstacle course comprising four obstructed-gait tasks set at three progressively challenging levels: stepping over an obstacle, foot targeting, stepping across an obstacle, and negotiating a raised surface. 
at $10 \%, 20 \%$, and $30 \%$ of each participant's leg length, except for the foot targeting, which was set at foot length plus 3,5, and $7 \mathrm{~cm}$ for Levels 1,2, and 3, respectively.

The step-over obstacle consisted of a 10 -mm-diameter plastic tube $1 \mathrm{~m}$ in length with each end resting on a stand of adjustable height (increments of $1 \mathrm{~mm}$ ). Light contact with the tube caused it to fall. The step-across and foot-targeting boundaries were delimited by pieces of white adhesive tape stuck to the floor. Wooden raised surfaces of $1 \mathrm{~m}^{2}$ in area were used for the raised-surface task. A helper was stationed adjacent to each obstacle to assist participants in the event of instability.

\section{OBSTACLE-COURSE TESTING PROTOCOL}

The OC testing was undertaken 1 week after 1-RM assessment at pre- and posttesting in order to minimize any effect of muscle soreness subsequent to strength testing. No verbal encouragement was provided. Participants were asked to undertake the course at their customary walking speed in order to mimic gait conditions encountered during real-life locomotion. Five practice and test trials were allowed at each level. A 1- to 2-min rest period was given after each trial. The order of presentation of the levels was counterbalanced across participants to minimize any learning and practice effects. Electronic timing gates placed at the start and end of the course recorded the time taken to complete the course at each level. In addition, electronic timing gates placed $3 \mathrm{~m}$ and $1.5 \mathrm{~m}$ before and after each station, respectively, determined the time taken to negotiate each station (Figure 1).

\section{INTERVENTION PROGRAM}

The EXP group underwent a 3-month progressive resistance-training program designed to increase muscle strength of the lower body major muscle groups. Custom-designed pin-loaded weight machines were used. The initial intensity and volume were set at $60 \%$ of 1-RM and two sets of eight repetitions, respectively. Intensity progressed to $75 \%$ of $1-\mathrm{RM}$ and volume to three sets of eight repetitions at the end of the training period. One-RM testing was undertaken fortnightly to adjust intensity accordingly. Rest periods of 1.5-2 min were allowed between sets and exercises.

\section{RELIABILITY OF THE DEPENDENT MEASURES}

Fourteen participants ( 7 men and 7 women) were selected to determine the reliability of the dependent measures. The method error of repeated measurements (ME\%) was used to establish intra- and interday variability. The interday reliability assessment occurred 1 week after the initial testing session. The ME\% method was determined using the following formula:

$$
\frac{\mathrm{ME}}{\left(\overline{\mathrm{x}_{1}}+\overline{x_{2}}\right) / 2} \times 100, \text { where } \mathrm{ME}(\mathrm{SD})=\sqrt{\frac{\sum(D-\bar{D})^{2}}{2(N-1)}}
$$

and $D=$ the difference between Trials 2 and 1 . 
From the 1-RM strength data collected, maximal force ( $\mathrm{kg}$ ) was defined as the heaviest successful weight lifted for hip flexion and extension, knee extension and flexion, and ankle plantar flexion. The intra- and interday ME\% scores for the 1-RM measures ranged from $4.7 \%$ to $15.7 \%$ and from $6.9 \%$ to $17.3 \%$, respectively. The mean times (s) to complete the course at each level and to negotiate each station of the $\mathrm{OC}$ were computed. The intraday $\mathrm{ME} \%$ values for the $\mathrm{OC}$ tasks ranged from $2.2 \%$ to $4.0 \%$. The interday $\mathrm{ME} \%$ scores were marginally higher, ranging from $2.5 \%$ to $4.5 \%$

\section{Statistical Analysis}

A one-way analysis of variance (ANOVA) was used to determine differences between the two groups at Week 0 (pretest) on the dependent measures. Repeatedmeasures ANOVAs were used to determine Group $\times$ Time interactions and between-group effects. Within-group changes (pre- to posttest) were investigated with a paired $t$ test using a Bonferroni adjustment. An alpha level of $p<.05$ was chosen as the criterion for significance. All statistical analyses were computed using the Statistics Package for Social Sciences (SPSS Version 10.0).

\section{Results}

\section{PARTICIPANTS}

One male participant dropped out of the EXP group for reasons unrelated to the study. The final sample ( $N=44 ; 29$ women, 15 men $)$ recorded a $M \pm S D$ age of $68.2 \pm$ 1.5 years (range 61-88), height of $165.7 \pm 1.7 \mathrm{~cm}$ (range 151-182), and body mass of $75.6 \pm 3.1 \mathrm{~kg}$ (range 47-115). The pretest characteristics for the CON $(n=15 ; 10$ women, 5 men) and $\operatorname{EXP}(n=29 ; 19$ women, 10 men) groups are shown in Table 1. A one-way ANOVA showed no significant between-group differences at pretest on any of these characteristics.

\section{1-RM}

There were no significant between-group differences initially for the 1-RM hipextension $(p=.32)$, hip-flexion $(p=.95)$, knee-extension $(p=.37)$, knee-flexion $(p=.26)$, and ankle plantar-flexion exercises $(p=.39)$. Repeated-measures ANOVA, however, revealed significant Group $\times$ Time interactions $(p<.001)$ at Week 12, such that the EXP group recorded substantially greater gains in all five strength measures (mean gain $=144.8 \%$ ) than those of the CON group (mean gain $=$ $18.2 \%$; Table 2). The absolute strength gains for the weight trainers ranged from $11.9 \mathrm{~kg}$ for the knee flexion to $32.7 \mathrm{~kg}$ for the hip extension, compared with 0.8 - and 7.8-kg increases for the same exercises reported by the CON group.

\section{OBSTACLE-COURSE GAIT MEASURES}

All participants completed the OC at the three prescribed challenge levels. The group $(N=44) M \pm S D$ obstacle height and width for the step-over, across, and 
Table 1 Characteristics of the Control and Experimental Groups at Week 0 $(M \pm S D)$

Participant characteristic

Control $(n=15 ; \quad$ Experimental $(n=29$;

10 women, 5 men) 19 women, 10 men) $\quad p^{\text {a }}$

Age (yrs)

$68.0 \pm 2.9$

$68.5 \pm 2.5$

.78

Height $(\mathrm{cm})$

$165.3 \pm 1.8$

$165.9 \pm 1.6$

.81

Body mass (kg)

$71.9 \pm 2.9$

$76.7 \pm 3.0$

.52

Leg length $(\mathrm{cm})$

$87.6 \pm 0.9$

$89.6 \pm 0.8$

.69

Foot length $(\mathrm{cm})$

$27.5 \pm 0.3$

$27.8 \pm 0.3$

.95

Hip extension $(\mathrm{kg})$

$24.2 \pm 3.7$

$25.9 \pm 2.8$

.76

Hip flexion (kg)

$13.7 \pm 1.8$

$13.8 \pm 1.5$

.94

Knee extension $(\mathrm{kg})$

$16.2 \pm 2.4$

$13.9 \pm 1.7$

.37

Knee flexion $(\mathrm{kg})$

$10.7 \pm 1.6$

$8.9 \pm 1.5$

.26

Ankle plantar flexion (kg)

$12.6 \pm 2.6$

$10.4 \pm 1.3$

.39

${ }^{a}$ Using one-way ANOVA.

Table 2 Strength Changes in the Control and Experimental Groups After 12 Weeks of Resistance Training $(M \pm S D)$

$\frac{\text { Control }}{\text { Change }(\mathrm{kg}) \quad \% \text { Change }} \quad \frac{\text { Experimental }}{\text { Change }(\mathrm{kg}) \quad \% \text { Change } \quad p^{\mathrm{a}}}$

Hip extension

$$
7.8 \pm 3.9 \quad 22.9 \pm 6.9^{\mathrm{b}}
$$

$32.7 \pm 3.6$

$132.9 \pm 6.9^{\mathrm{b}}$

$<.001$

Hip flexion

$2.3 \pm 1.6$

$16.2 \pm 4.9$

$17.2 \pm 1.9$

$124.6 \pm 5.2^{\mathrm{b}, \mathrm{c}}$

$<.001$

Knee extension

$5.2 \pm 2.2$

$32.7 \pm 5.6^{\mathrm{b}}$

$17.9 \pm 3.2$

$130.6 \pm 4.9^{\mathrm{b}}$

$<.001$

Knee flexion

$0.8 \pm 1.2$

$7.1 \pm 3.6$

$11.9 \pm 2.6$

$188.9 \pm 2.8^{\mathrm{b}, \mathrm{c}}$

$<.001$

Plantar flexion

$1.4 \pm 2.2 \quad 11.9 \pm 4.1$

$15.3 \pm 2.9$

$147.1 \pm 5.6^{\mathrm{b}}$

$<.001$

a Using repeated-measures ANOVA Group $\times$ Time interaction. ${ }^{\mathrm{b}}$ Denotes a significant time effect $(p<.05)$. ${ }^{c}$ Denotes a significant group effect $(p<.05)$.

raised-surface tasks were $8.9 \pm 0.4,17.5 \pm 0.9$, and $26.6 \pm 1.3 \mathrm{~cm}$ at Levels 1,2 , and 3 respectively. The group mean foot lengths for the targeting task at Levels 1, 2, and 3 were $34.8 \pm 1.6,32.8 \pm 1.6$, and $30.8 \pm 1.6 \mathrm{~cm}$, respectively. The obstacle-crossing times at pretest, shown in Table 3 , increased as a function of the challenge levels, irrespective of group.

There were no significant differences between the two groups at Week 0 for the times to negotiate the step-over $(p=.133)$, raised-surface $(p=.138)$, step-across 


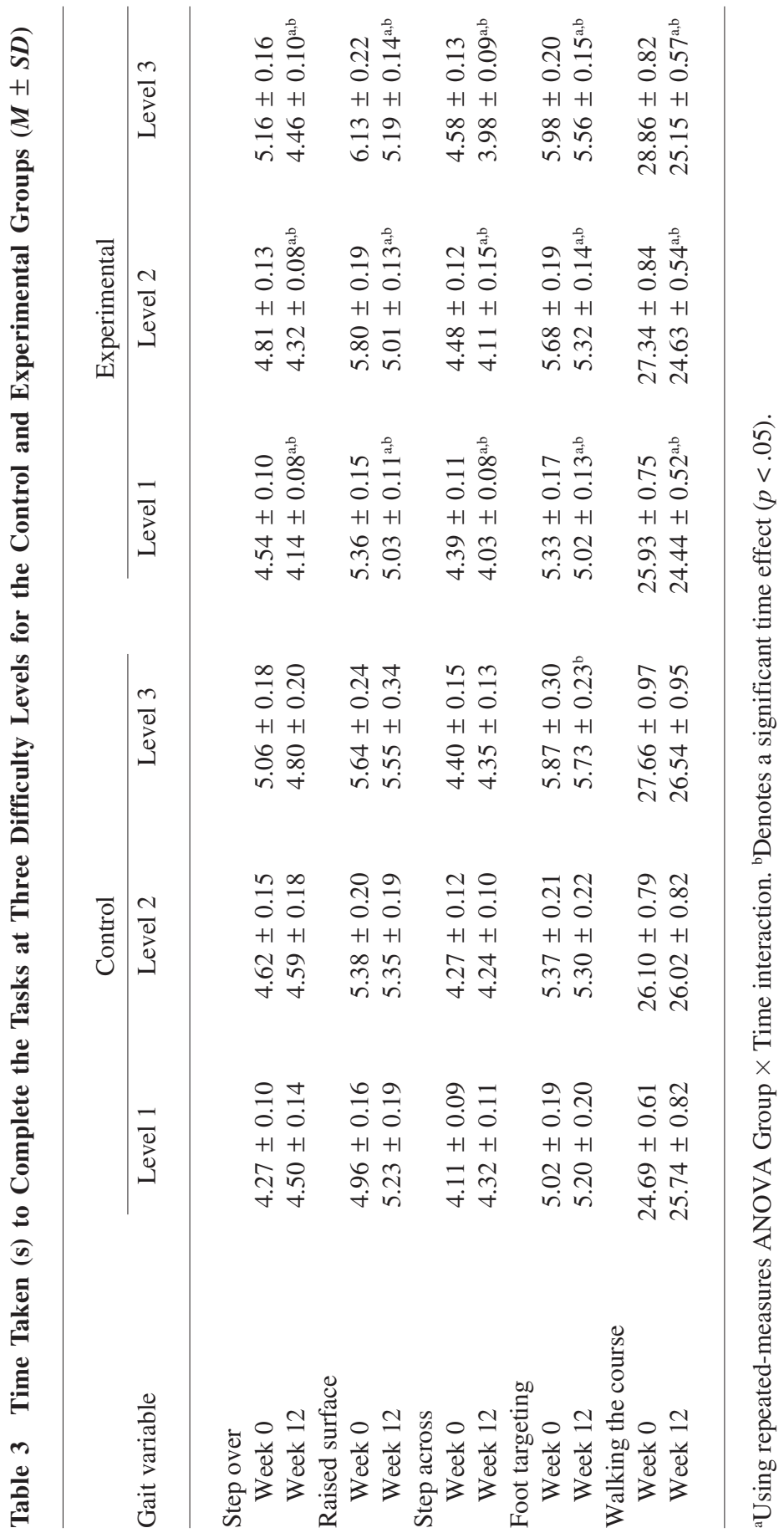


( $p=.113)$, and foot-targeting tasks $(p=.3)$ and to walk the course $(p=.331)$. After training, statistical analyses revealed significant Group $\times$ Time interactions $(p<$ .001 ) on the five OC dependent variables, such that the EXP group significantly reduced its crossing time (averaged for the three levels) by $10.8 \%( \pm 1.4 \%), 11.7 \%$ $( \pm 1.3 \%), 9.8 \%( \pm 1.4 \%), 9.6 \%( \pm 1.2 \%)$, and $9.5 \%( \pm 1.3 \%)$ for the step-over, raised-surface, step-across, and foot-targeting conditions and to walk the course, respectively $(p<.05$; Table 3$)$. In contrast, the performance of the CON group remained unchanged overall except at Level 3 for the foot-targeting condition, in which a significant decrease in crossing time was recorded $(p=.03)$.

As shown in Figure 2, the percentage change calculated from Weeks 0 to 12 shows that only the EXP group consistently improved its performance, displaying substantially higher improvement rates than the $\mathrm{CON}$ group at all levels for the five gait tasks. Figure 2 also shows that the magnitude of the percentage change recorded by the EXP group consistently increased as a function of the challenge level of task.

\section{Discussion}

This is the first study to directly assess the effect of improved leg strength on obstructed-gait tasks as commonly encountered by community-living older adults. The primary outcome of the present investigation was that 12 weeks of progressive resistance training significantly improved dynamic lower body strength and walking speed over on an $\mathrm{OC}$ at three progressively challenging levels. Given that most falls in the elderly occur when they are transferring from one support surface to another and negotiating obstacles (Grabiner \& Enoka, 1995; Sparrow, Shinkfield, Chow, \& Begg, 1996), that 47\% result from tripping over an object (Overstall, Exton-Smith, Imms, \& Johnson, 1977), and that the largest proportion of falls in public places (involving elderly individuals) occurs while negotiating an obstacle (Simoneau, Cavanagh, Ulbrecht, Leibowitz, \& Tyrrell, 1991), the findings of the present study demonstrate that enhanced lower body muscle strength improves functional gait performance in community-dwelling older adults.

The major lower body muscle groups play an important role in the smooth forward translation of the center of mass when increased stress is imposed on the musculoskeletal system during obstructed gait (Chou \& Draganich, 1998; Patla \& Rietdyk, 1993). In addition, leg weakness has been associated with impaired function, shown by decreased walking speed (Judge, Underwood, \& Gennosa, 1993). The substantial gains in the 1-RM of the major leg muscles of the EXP participants were accompanied by significant improvements in crossing speed in all obstructed tasks. Previous research has reported the positive effects of improved strength on unobstructed-gait speed (Chandler et al., 1998; Fiatarone et al., 1994; Hunter et al., 1995), and the present findings confirm and extend previous proposals concerning the benefits of enhanced lower body strength on functional mobility in community-living older adults.

The critical role of enhanced strength in complex locomotor tasks is further emphasized in this study-superior improvements in crossing times were consistently recorded at the two highest levels of the OC compared with Level 1 (Figure 2 ). It is argued that at the highest challenge levels, greater demands are placed on the neuromuscular system, such as those associated with increased stride length, greater limb elevation, and higher obstacle clearance. Consequently, enhanced 


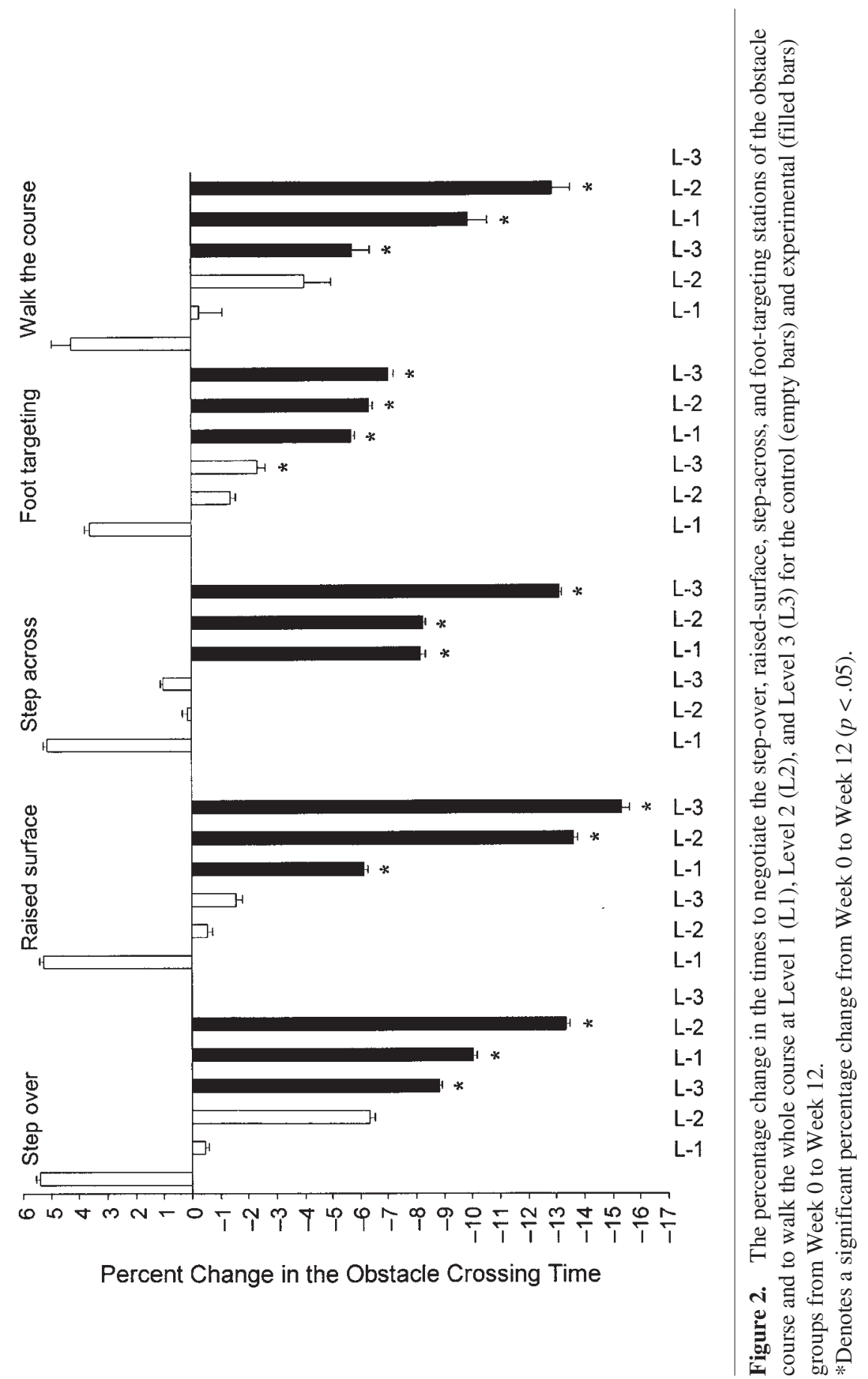


strength in the EXP group could have conferred a greater potential for improvement in the obstructed-gait tasks set at the highest levels than at the lower level. Nonetheless, it is also possible that muscle strength is not a limiting factor in gait tasks set at the lowest level, especially in healthy community-living older adults. For example, Cress, Conley, Balding, Hansen-Smith, and Konczak (1996) found that the contribution of improved isokinetic strength was more critical at the two highest riser heights $(36$ and $47 \mathrm{~cm})$ than at the lowest $(24 \mathrm{~cm})$ in healthy older women between 65 and 83 years of age.

The systematic increase in crossing time as a function of the progressively challenging levels demonstrates that the OC used in this study was sensitive to the stratification of the gait tasks. The relatively small $\mathrm{ME} \%$ recorded in the study also showed that a progressively challenging $\mathrm{OC}$ is a reliable measure to assess gait performance in that population. Because the percentage gains found in the EXP group on the OC dependent variables were superior to the ME percentages, it can be concluded that the improvements in crossing times were not a result of variation in measurement methodology.

Although significant improvements were found in all gait tasks, the foottargeting condition recorded the smallest percentage gain. It is possible that the foottargeting task challenges not only the strength of the postural muscles but also the balance-control system. Targeting requires longer single-foot stance time to effectively place the lead foot in a more restricted area. It is possible that although resistance training improves the ability to undertake obstacle-crossing tasks, it might have limited effects on the neuromuscular processes associated with precise lower limb control and balance. Further research is needed to confirm this hypothesis.

In the present randomized-controlled study, 12 weeks of progressive resistance training produced substantial increases in lower body strength in the EXP group. Comparable strength gains have been reported in older men and women using similar resistance-training programs (Charette et al., 1991; Frontera, Meredith, O'Reilly, Knuttgen, \& Evans, 1988). Our results confirm that a systematic resistancetraining program of moderate to high intensity can produce substantial and rapid strength improvement in older adults. It is important to note that, in addition to the positive effects of improved strength on obstructed gait found in this study, strength gains might be functionally important. Intervention studies have shown that increased strength can improve the functional status of older adults in activities such as stair climbing, chair rising, stooping, and spontaneous physical activity (Chandler \& Hadley, 1996; Fiatarone et al., 1994; Fisher, Pendergast, \& Gresham, 1991). It has also been suggested that 10-20\% increase in quadriceps strength might delay the threshold of dependency for up to 2 decades in older adults (Young, 1986). The evidence of functional improvement subsequent to resistance training is promising, and further research is warranted to accurately determine the effect of improved strength on everyday motor performance.

Finally, it should be emphasized that the findings of the present study are viewed within two specific parameters. First, the gait tasks were performed at comfortable or customary walking speed in order to simulate community locomotion. Although it could be speculated that similar gains could have been anticipated using maximal speed because positive correlations between maximal walking speed and lower body strength have been found in older adults (Fiatarone et al., 1990; 
Rantanen, Era, \& Heikkinen, 1994), future investigations are needed to confirm the gait improvements recorded in the present study but using maximal speed. Second, a 1-RM strength method was used in the current study because it was hypothesized that it would better mimic the type of dynamic muscle contractions produced during obstacle negotiation than would isometric or isokinetic strength assessment and would therefore improve training and testing specificity. Future research is warranted to determine whether the gait improvements recorded in this study could be obtained using other forms of strength assessment.

In conclusion, the findings of this study showed that a progressive 12 -week resistance-training program produced significant strength gains in the leg strength of community-living older adults. Enhanced strength significantly improved the obstacle-crossing times as assessed on an obstacle course set at three progressively challenging levels replicating a range of environmental challenges. Given that over half of falls in older adults occur during obstacle negotiation and that falls are costly and a major cause of serious soft-tissue injuries, fractures, morbidity, and institutionalization(Rubenstein \& Josephson, 1992; Sparrow et al., 1996; Tinetti, Speechley, \& Ginter, 1988), the findings of the present study are significant not only in terms of enhanced functional mobility but also as a falls-prevention strategy. With the inevitable declines in muscle strength and mass that accompany aging, exerciseinduced strength gains could be a long-term, cost-effective alternative to help community-living older adults remain functionally mobile and, possibly, reduce their vulnerability to a trip or a fall during obstacle negotiation.

\section{References}

Brown, M., Sinacore, D., \& Host, H. (1995).The relationship of strength to function in the older adult. Journals of Gerontology: Series A, 50A(special issue), 55-59.

Chandler, J., Duncan, P., Kocherberger, G., \& Studenski, S. (1998). Is lower extremity strength gain associated with improvement in physical performance and disability in frail, community-dwelling elders? Archives of Physical Medicine and Rehabilitation, 79, 24-30.

Chandler, J., \& Hadley, H. (1996). Exercise to improve physiologic and functional performance in old age. Clinics in Geriatric Medicine, 14, 761-784.

Charette, S., McEnvoy, L., Pyka, G., Snow-Harter, C., Guido, D., Wiswell, R., \& Marcus, R. (1991). Muscle hypertrophy response to resistance training in older women. Journal of Applied Physiology, 70, 1912-1916.

Chou, L., \& Draganich, L. (1998). Increasing obstacle height and decreasing toe-obstacle clearance affect the joint moments of the stance limb differently when stepping over an obstacle. Gait and Posture, 8, 186-204.

Cress, M.E., Conley, K.E., Balding, S.L., Hansen-Smith, F., \& Konczak, J. (1996). Functional training: Muscle structure, function, and performance in older women. Journal of Orthopaedic and Sports Physical Therapy, 24(1), 4-10.

Fiatarone, M., Marks, E., Ryan, N., Meridith, C., Lipsitz, L., \& Evans, W. (1990). High intensity strength training in nonagenarians. Journal of the American Geriatrics Society, 263, 3029-3034.

Fiatarone, M., O’Neill, E., Ryan, N., Clements, K., Solares, G., Nelson, M., et al. (1994). Exercise training and nutritional supplementation for physical frailty in very elderly people. New England Journal of Medicine, 330, 1769-1775. 
Fisher, N., Pendergast, D., \& Gresham G. (1991). Muscle rehabilitation: Its effects on muscular and functional performance on patients with knee osteoarthritis. Archives of Physical Medicine and Rehabilitation, 72, 367-374.

Frontera, W., Meredith, C., O’Reilly, K., Knuttgen, H., \& Evans, W. (1988). Strength conditioning in older men: Skeletal hypertrophy and improved muscle function. Journal of Applied Physiology, 64, 1038-1044.

Grabiner, M.D., \& Enoka, R.M. (1995). Changes in movement capabilities with aging. Exercise and Sport Sciences Reviews, 23, 65-104.

Hunter, G., Treuth, M., Weinsier, R., Szabo, T., Kell, S., Roth, D., \& Nicholson, C. (1995). The effects of strength conditioning on older women's ability to perform daily tasks. Journal of the American Geriatrics Society, 43, 756-760.

Imms, F., \& Eldholm, O. (1981). Studies of gait and mobility in the elderly. Age and Ageing, 10, 147-156.

Judge, J., Underwood, M., \& Gennosa, T. (1993). Exercise to improve gait velocity in older persons. Archives of Physical Medicine and Rehabilitation, 74, 400-406.

Means, K. (1996). The obstacle course: A tool for the assessment of functional balance and mobility in the elderly. Journal of Rehabilitation Research and Development, 33, 413428.

Means, K., Rodell, D., \& O'Sullivan, P. (1996). Use of an obstacle course to assess balance and mobility in the elderly: A validation study. American Journal of Physical Medicine and Rehabilitation, 75, 88-95.

Morganti, C., Nelson, M., Fiatarone, M., Dallal, G., Economos, C., Crawford, B., \& Evans, W. (1995). Strength improvement with $1 \mathrm{yr}$ of progressive resistance training in older women. Medicine and Science in Sports and Exercise, 27, 906-912.

Overstall, P.W., Exton-Smith, A.N., Imms, F.J., \& Johnson, A.L. (1977). Falls in the elderly related to postural imbalance. British Medical Journal, 1(6056), 261-264.

Patla, A., \& Rietdyk, S. (1993). Visual control of limb trajectory during locomotion: Effect of obstacle height and width. Gait and Posture, 1, 45-60.

Rantanen, T., Era, P., \& Heikkinen, E. (1994). Maximal isometric strength and mobility among 75-year-old men and women. Age and Ageing, 23, 132-137.

Roman, W., Fleckenstein, J., Stray-Gundersen, J., Alway, S., Peshok, R., \& Gonyea, W. (1993). Adaptations to the elbow flexors of elderly males after heavy-resistance training. Journal of Applied Physiology, 72, 750-754.

Rubenstein, A., \& Josephson, K. (1992). Causes and prevention of falls in elderly people. In B. Vellas, M. Toupet, L. Rubenstein, J.L. Albarede, \& Y. Christens (Eds), Falls, balance and gait disorders in the elderly (pp. 21-38). Paris: Elsevier.

Simoneau, G.G., Cavanagh, P.R., Ulbrecht, J.S., Leibowitz, H.W., \& Tyrrell, R.A. (1991). The influence of visual factors on fall-related kinematic variables during stair descent by older women. Journal of Gerontology, 46(6), M188-M195.

Sparrow, W., Shinkfield, A., Chow, S., \& Begg, R. (1996). Characteristics of gait in stepping over obstacles. Human Movement Science, 15, 605-622.

Tinetti, M., Speechley, M., \& Ginter, R. (1988). Risk factors for falls among elderly persons living in the community. New England Journal of Medicine, 319, 1701-1707.

Young, A. (1986). Exercise physiology in geriatric practice. Acta Medica Scandinavica, 711(suppl), 227-232. 
